\title{
Minimally Invasive Subpericranial Model: Can It Be Used to Study Bone Substitutes?*
}

\author{
Dror M. Allon ${ }^{1 \#}$, Irit Allon ${ }^{2}$, Yakir Anavi ${ }^{1}$ \\ ${ }^{1}$ Department of Oral \& Maxillofacial Surgery, Rabin Medical Center, Affiliated with the Sackler School of Medicine, \\ Tel Aviv University, Petach Tikva, Israel \\ ${ }^{2}$ Department of Oral Pathology and Oral Medicine, School of Dental Medicine, Tel Aviv University, Tel Aviv, Israel \\ Email: \#drorallon@gmail.com
}

Received October 31, 2012; revised December 2, 2012; accepted December 11, 2012

\begin{abstract}
Objective: To evaluate the feasibility and effectiveness of a new approach for craniofacial augmentation by a minimally-invasive subpericranial injection. Three commercially available semi-liquid bioceramic bone-grafting materials were examined for this application in a rat model. Material and Methods: Twenty-four adult male rats were randomly assigned to undergo onlay calvarial grafting by subpericranial injection of three semi-liquid bone void fillers: Norian SRS $^{\circledR}$ (calcium phosphate), ChronOs Inject ${ }^{\mathrm{TM}}$ (porous beta-tricalcium phosphate), or BonePlast ${ }^{\circledR}$ (calcium $^{-1}$ sulfate). Two rats in each group were harvested after 24 hours to serve as controls. The rest were studied after 16 weeks. The volume fraction of the following parameters was morphometrically measured: new bone, blood vessels, residual bone filler and inflammation. Results: In all study groups (including controls), histological examination demonstrated that bone fillers were successfully delivered to the desired subpericranial space by the percutaneous injection method. New bone formation was evidenced adjacent to the cranial bone in all the study groups. The Norian filler material survived in a significantly higher volume fraction $(38.4 \% \pm 6.5 \%)$ than the ChronOs filler $(18.8 \% \pm 1.6 \% ; \mathrm{P}<0.0001)$ and the BonePlast filler $(17.8 \% \pm 1.5 \% ; \mathrm{P}<0.0001)$. New bone was formed in all groups, particularly adjacent to the interface of graft material with native bone but only to minimal extent. Conclusion: This new approach for craniomaxillofacial augmentation was successfully demonstrated in a rat model. The Norian filler (calcium phosphate) demonstrated superior space preservation abilities. This model may be further applied to test new injectable bone substitutes in the craniomaxillofacial area.
\end{abstract}

Keywords: Subpericranial Approach; Bone Substitute; Subperiosteal Injection; Augmentation; Bone Regeneration

\section{Introduction}

Facial bone defects caused by trauma or tumors pose a reconstruction challenge for oral and maxillofacial surgeon and plastic surgeon. The absence of bone segments in the facial skeleton is traditionally managed by replacing the defect with autogenous bone. However, the procedure may be associated with soft-tissue complications, including unaesthetic surgical scars and donor-site morbidity. Although synthetic bone fillers offer a potential alternative to autogenous bone grafts, extensive efforts to develop an ideal alloplastic bone substitute have so far been unsuccessful [1-4]. Given the complex structure of the craniofacial skeleton, the graft material must ideally be biocompatible and resilient, fast-setting, easily inserted, and able to be molded and integrate with surrounding bone in order to mimic the original bone structure [5-8].

\footnotetext{
"Conflict of interest: the authors declare no conflict of interest.

${ }^{\#}$ Corresponding author.
}

Osteoconductive materials are intended to serve as a scaffold for new bone formation by capillary in growth and invasion of osteoprogenitor cells from the recipient bed [9]. Even with the emerging use of osteoprogenitor cells or osteoinduction molecules there is still ample need of bone void filler materials. Several reviews describing the different subgroups of injectable bone substitute materials developed to date have been published [10-12].

Since the periosteum contains subsets of progenitor cells that have the ability to differentiate into osteoblasts by diverse physical or chemical stimulation, such as bone fracture, inflammation, and tumor development, taking advantage of this property sounds an interesting attitude. It was already suggested that bone repair proceeds from the periosteum on the outer and inner surfaces adjacent to the bony defect [13].

The aim of the present study was to examine the feasibility and effectiveness of a novel minimally invasive 
approach, in which injection of semi liquid bone graft material was directed transcutaneously to a subpericranial plane, immediately adjacent to the cranial bone in a rat model. Three commercially available bone void fillers (BVF) were compared by microscopic examination, 16 weeks post grafting, by evaluating the volume fraction of new bone, bone filler material, blood vessels and inflammation.

\section{Materials and Methods}

\subsection{Study Groups and Procedure}

The study was approved by the Committee for Ethics in Animal Experiments of Rabin Medical Center. Twentyfour Fischer inbred isogenic adult male rats, each weighing 450 - 500 grams, were randomly divided to three groups. Each group assigned to undergo subpericranial onlay calvaria grafting with one of three BVFs: Group 1 Norian Skeletal Repair System ${ }^{\circledR}$ (SRS; calcium phosphate; Synthes, Solothurn, Switzerland) $(n=8)$, Group 2 - ChronOs Inject ${ }^{\mathrm{TM}}$ (porous beta-tricalcium phosphate; Synthes) $(n=8)$, and Group 3-BonePlast ${ }^{\circledR}$ (calcium sulfate; Biomet Osteobiologics, Parsippany, NJ) $(\mathrm{n}=8)$. All three materials were ready for use in injectable form. All rats were anesthetized with an intraperitoneal injecttion of $4 \mathrm{mg} / \mathrm{kg}$ chloral hydrate, and the cranial site was scrubbed with $10 \%$ povidone-iodine solution. Thereafter, $1000 \mathrm{~mm}^{3}$ of the assigned BVF graft material were injected transcutaneously via a beveled cannula $(2 \mathrm{~mm}$ diameter) into the subpericranial plane in a closed fashion to the cranial bone. The needle was introduced through the skin and subcutaneous tissue until it reached the solid outer cranial bone table. With the needle bevel parallel to the bone surface the injectable material was applied in a controlled pressure to achieve periosteal separation by the tumescent effect of the hydraulic pressure of the semi liquid material. The material was left to set, without pressure. Neither local anesthesia nor vasoconstrictors were used at the surgical site.

Postoperatively, all rats were housed in stainless steel hutches maintained at a temperature of $19^{\circ} \mathrm{C}-25^{\circ} \mathrm{C}$, in ventilated air of approximately $55 \%$ humidity. They were fed standard rat chow with water ad libitum. An elixir of acetaminophen with codeine was added to the drinking water during the first three postoperative days, and antibiotics were added for the first five days.

Two rats from each of the 3 groups (total 6 rats) were sacrificed $24 \mathrm{~h}$ after surgery in a carbon dioxide chamber to serve as the control group (fresh graft after full setting, with no osteoblastic activity expected). The other 18 rats were scarified 16 weeks later in the same manner. Onlay grafts were harvested along with supporting cranial bone and overlying scalp. The specimens were maintained in $4 \%$ buffered formalin.
The rat skulls were sawed, and the top part (skull cap) was incubated in decalcifying solution ( $10 \%$ formic acid) at room temperature for $7-8$ days, followed by washing under running tap water for 15 min. The skull caps were then cut parallel to the coronal plane, placed in plastic cassettes, dehydrated by an automated machine, embedded in paraffin block, and sectioned at approximately 5 microns. Sections were placed on glass slides and incubated at $82^{\circ} \mathrm{C}$ for $30 \mathrm{~min}$. The slides were stained with Hematoxylin and Eosin ( $\mathrm{H} \& \mathrm{E})$ using an automated machine and with Masson trichrome (Bio Optica, Milan, Italy) according to the manufacturer's instructions.

\subsection{Histological Analysis}

Each slide was evaluated macroscopically and microscopically.

The macroscopic measurements included calculations of the relative residual volume of BVF at the end of the study period. Since the total injected volume was 1000 $\mathrm{mm}^{3}$, and since the outcome was very similar to a semi hemisphere, measurements were conducted by the formula: volume $=\left[4 \pi r^{3} / 3 \times 1 / 2\right] / 1000 \times 100 \quad($ Figure 1) .

The microscopic measurements consisted of a morphometric analysis of the volume fraction $\left(\mathrm{V}_{\mathrm{v}}\right)$ of the following parameters: newly formed bone, newly formed blood vessels, residual BVF grafting material; chronic inflammation. The morphometric analysis was conducted as previously described 1 , briefly, the entire slide was screened at low magnification $(\times 40)$, and the region of interest (ROI) identified as the implantation area. The entire area was assessed using the modified point counting method 1. At $\times 100$ magnification, each standard field defined by a 100-square grid (Olympus, Tokyo, Japan) with a surface of $0.64 \mathrm{~mm}^{2}$, comprising 121 intersections between each horizontal and vertical line, was mounted on a $\mathrm{BH} 2$ Olympus microscope. The number of intersections containing new bone, blood vessels, bone filler material and inflammation were counted: when an intersection hit one of these elements, the specified element scored one point. The intersections were counted in each section, and consecutive fields covered the entire ROI.

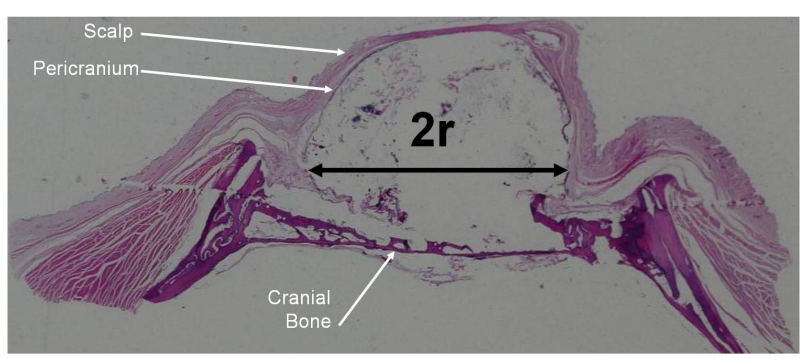

Figure 1. Measurement method of the macroscopic preserved volume after 16 weeks $\left(v=\left[4 \pi r^{3} / 3 \times 1 / 2 / 1000\right] \times 100\right)$. $(r$, radii of the calculated hemisphere in the tissue section presenting the largest hemisphere). 
Then, the sum of points overlying each specified element was calculated, divided by the total number of counted intersections in that section and multiplied by 100 . This was defined as the volume fraction $\left(\mathrm{V}_{\mathrm{v}}\right)$ of each element. A matched Masson trichrome staining helped to locate new ossification sites and was particularly useful for interpreting equivocal cases.

\subsection{Statistical Analysis}

Differences in the mean $\mathrm{V}_{\mathrm{v}}$ of the various elements among and between the study groups were statistically analyzed using one-way ANOVA. Statistical significance was set at $\mathrm{P}<0.05$. The statistical package Instat was used for computations.

\section{Results}

\subsection{Macroscopic Observation}

All animals recovered well after surgery. No macroscopic infection of the wounds was noted. There were no side effects, such as paralysis, convulsions, respiratory distress or signs of pain.

\subsection{Histological Analysis}

All the study groups including the controls, showed presence of bone void filler material in the subpericranial plane immediately adjacent to the cranial bone as expected. In all the three study groups, new bone was evidenced upon the external cranial table with no significant differences between the groups (Figures 2(a) and (c)). Bone formation was accompanied by angiogenic activity (Figures 3(a) and (b)), with minimal inflammation. Sixteen weeks after implantation, some areas of bone neoformation were already replaced by mature bone.

\subsection{Macroscopic Calculation}

The Norian group was much more capable to maintainthe volume $(41.9 \% \pm 6.9 \%)$ (Figure 1) compared to the ChronOs group $(20.9 \% \pm 2 \% ; \mathrm{P}=0.004)$ or to the BonePlast group $(19.8 \% \pm 1.84 \% ; \mathrm{P}=0.04)$. The preserved volume the controls of each group (sacrificed day 1) were expectedly much higher $(83.7 \%+21 \%, 82 \%+$ $1.2 \%$ and $84.7 \%+6.4 \%$ for the controls of Norian, ChronOs and BonePlast, respectively; $\mathrm{P}<0.0001$ for all).

\subsection{Morphometric Analysis}

The results of the morphometric analysis are presented in Table 1.

In each of the controls filler was abundant but neither new bone nor blood vessels were noted. When compareing between the different groups, Norian showed highermean $\mathrm{V}_{\mathrm{v}}$ of filler presence $(38.4 \%+6.5 \%)$ than Chron-Os $(18.8 \%+1.6 \%, \mathrm{P}<0.0001)$ and BonePlast

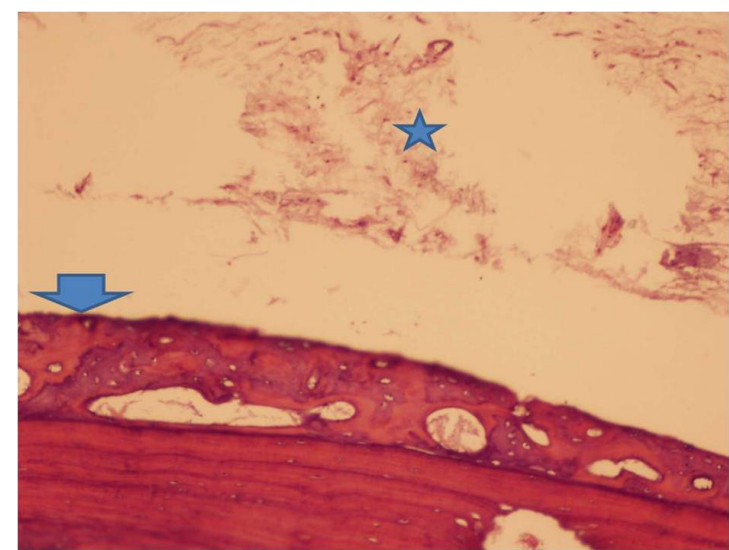

(a)

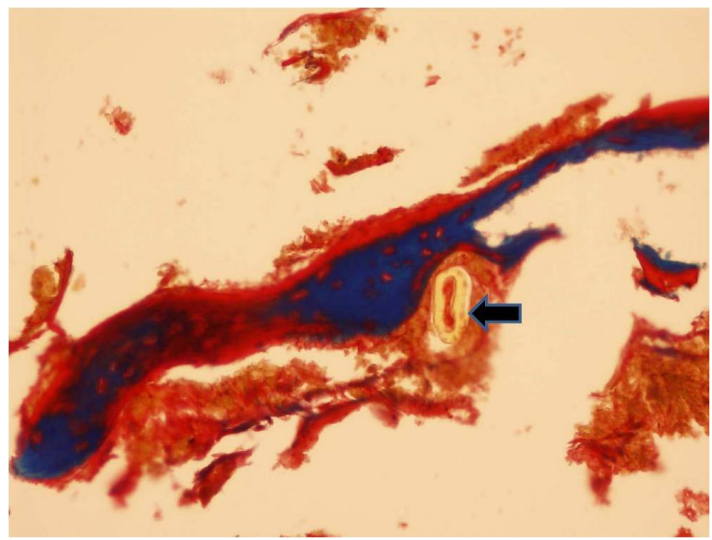

(b)

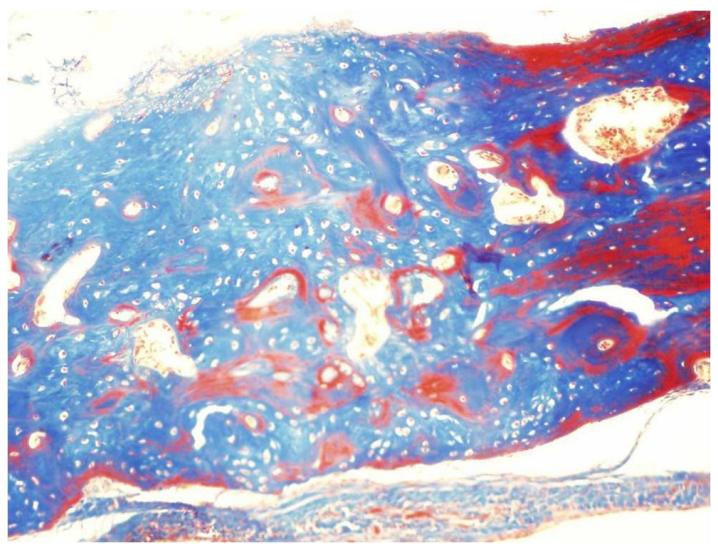

(c)

Figure 2. Formation of new bone in the study groups: (a) Woven bone (arrow) formed adjacent to existing lamellar bone and in close proximity to ChronOs remnants (star) (H \& $\mathrm{E} \times 200$ magnification); (b) Woven bone in blue adjacent to Norian circular remnants (arrow) (Masson trichrome $\times$ 100 magnification); (c) In the ChronOs group difference is noted between the regular native bone on the right side, where the compact bone is localized in the periphery (red), and the woven bone in the middle (blue); on the left side, where bone neoformation occurs, the process is characterized by woven (blue) bone area intermingled with mature (red) areas (Masson trichrome staining, $\times 200$ magnification). 
Table 1. Mean volume fraction $\left(V_{v}\right) \pm S D$ of new bone, blood vessels, bone void filler material and inflammation. The Norian is found to function as a better space maintainer and presents higher new bone formation. New bone is built only to a limited extent.

\begin{tabular}{ccccc}
\hline Inflammation & $\begin{array}{c}\text { Bone filler } \\
\text { material }\end{array}$ & $\begin{array}{c}\text { Blood } \\
\text { vessels }\end{array}$ & $\begin{array}{c}\text { New } \\
\text { bone }\end{array}$ & $\begin{array}{c}\text { Type of } \\
\text { bioceramic } \\
\text { filler }\end{array}$ \\
\hline $6.4 \pm 1.8$ & $38.4 \pm 6.5^{1}$ & $14.7 \pm 6.5$ & $7.8 \pm 1.7$ & $\begin{array}{c}\text { Norian } \\
\text { Skeletal } \\
\text { Repair } \\
\text { System }\end{array}$ \\
$7.5 \pm 1.1$ & $18.8 \pm 1.6^{1,2}$ & $16.3 \pm 5.7$ & $6.9 \pm 2.3$ & $\begin{array}{c}\text { ChronOs } \\
\text { Inject }^{\mathrm{TM}}\end{array}$ \\
$6.9 \pm 3$ & $17.8 \pm 1.5^{1,2}$ & $12 \pm 2.6$ & $7.0 \pm 2$ & BonePlast $^{\circledR}$ \\
\hline
\end{tabular}

${ }^{1} \mathrm{P}<0.0001$ relates to Norian vs ChronOs and Norian vs BonePlast; ${ }^{2} \mathrm{P}>$ 0.05 between ChronOs and BonePlast.

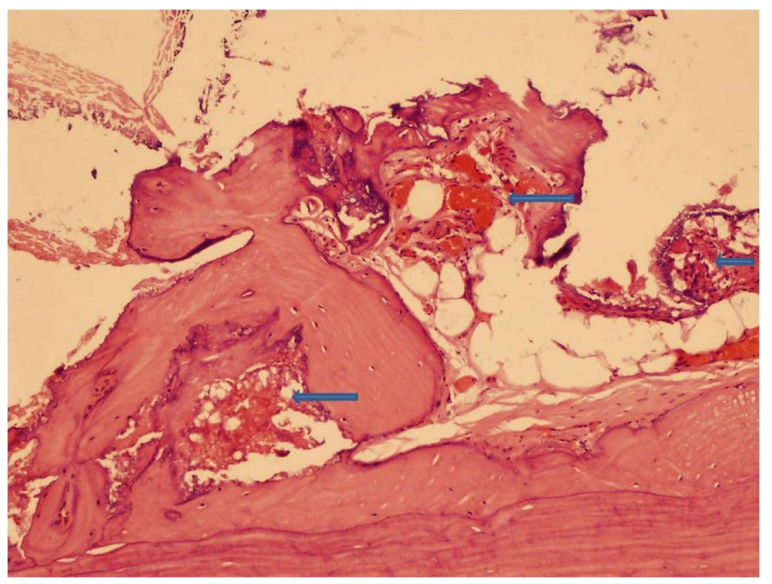

(a)

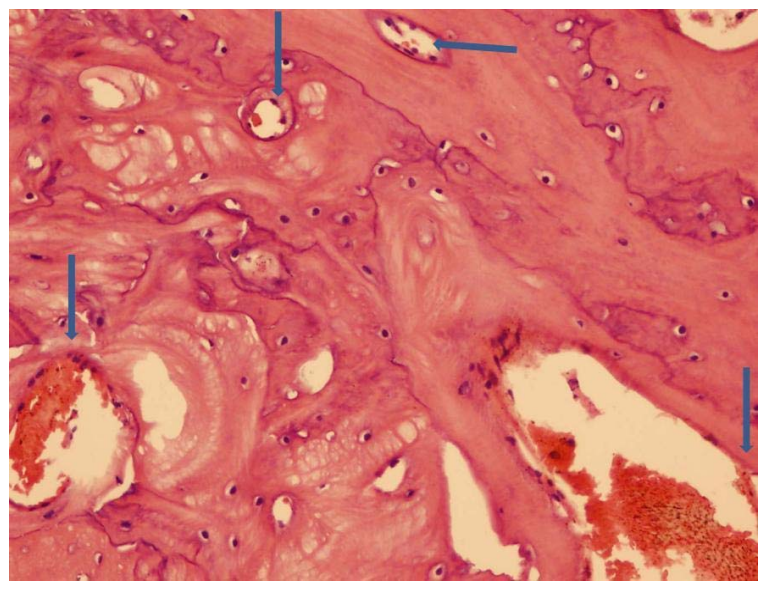

(b)

Figure 3. Osteogenic activity is accompanied by angiogenesis (arrows). (a) Norian (H \& $\mathrm{E} \times 100$ magnification); and in (b) BonePlast (H \& E $\times 200$ magnification).

$(17.8 \%+1.5 \%, \mathrm{P}<0.0001)$. Newly formed blood vessels were noted with no significant difference between the study groups. Inflammation was found to be minimal.

\section{Discussion}

This study presents a new approach to bone grafting, in which a subperiosteal injection of semi liquid bone osteoconductive materials is applied. Subperiosteal injection of bone filler for the reconstruction and augmentation craniofacial deformities has several advantages over open-flap procedures. It eliminates the need for exposure, thereby reducing the risk of graft contamination by skin or mucosal flora. The intact periosteal coverage of the graft maximizes its perfusion and osteogenic potential, as opposed to either covering the bone graft or bone substitute with a surgically-cut and therefore injured periosteal or mucoperiosteal flap or placing a barrier, such as guided bone regeneration (GBR) membrane, between the graft and the periosteum. From a biomechanical point of view, the uncut periosteum is able to withstand much higher tension forces over the augmented area, thus preventing dehiscence and other complications (including partial or total loss of the graft). Scar formation, which is a consideration in cosmetic facial augmentation procedures, is avoided completely as well. Additionally, injectable bone graft fillers eliminate the risk of infection and other complications at the bone-donor site and require less postoperative care. This is also applicable in children, in whom large amounts of autogenous bone may be difficult to harvest. In reconstruction and augmentation of the craniofacial skeleton, liquid bone grafts may be particularly applicable in mandibular reconstruction, augmentation of the ramus and symphysis, midfacial augmentation of the malar, paranasal, and maxillary regions, as well as supraorbital rim and frontal bone. The presented minimally-invasive surgical approach in a rat model, demonstrated new bone formation and filler presence in the subperiosteal plane, rendering this transcutaneous subperiosteal injection as a feasible model for studying the effectiveness of bone substitute materials in the craniofacial area: Firstly, the subperiosteal injection delivered the filler material to the right surgical plane. In all our samples the injected material was found to be located directly upon the cranial bone and underneath the periosteum/pericranium. This means that the subperiostal injection delivered the material to the right place, without opening the tissue with a flap. Secondly, the delivered injected material survived in place for four months. Being semi liquid, the question of whether new bone formation really occurs intrigued us. This study showed that although new bone is formed, it is rather limited and occurs particularly at the interface. The Norian presented superior space maintaining abilities compared to ChronOs and BonePlast. The $\mathrm{V}_{\mathrm{v}} \mathrm{s}$ of new bone were similar between the materials studied and comparable to the unfilled controls, described, for example, by Park et al. (2009), and lower of the $\mathrm{V}_{\mathrm{v}}$ of Bio-oss, hydroxy apetite and calcium sulfate hydroxyl apetite filled defects in the 
same study, although the last were studied earlier, in week 12 [14]. This points that the described mod is feasible and promising, other new bone substitutes that can be injected need to be sought.

In conclusion, this new presented transcutaneous subpericranial model was found feasible and able to test the osteoconductive properties in rat calvaria. This transcutaneous subpericranial model can be submitted to test further osteogenic and angiogenic properties of injectable and other bone substitutes.

Future directions may include testing other osteoconductive materials that can be injected subperiosteally and that may present better results in terms of bone formation; using materials that are able to change their semi liquid nature once injected and become porous, thus allowing bone osteoprogenitor cells and molecules to creep in and adding osteoinductive elements to the semi liquid osteoconductive materials.

\section{Acknowledgements}

This study was supported (in part) by grant no. 3-000005094 from the Chief Scientist Office of the Ministry of Health, Israel.

\section{REFERENCES}

[1] B. J. Costello, G. Shah, P. Kumta and C. S. Sfeir, "Regenerative Medicine for Craniomaxillofacial Surgery," Oral and Maxillofacial Surgery Clinics of North America, Vol. 22, No. 1, 2010, pp. 33-42. doi:10.1016/j.coms.2009.10.009

[2] M. H. Guskuma, E. Hochuli-Vieira, F. P. Pereira, I. Rangel-Garcia Jr., R. Okamoto, T. Okamoto and O. Magro Filho, "Bone Regeneration in Surgically Created Defects Filled with Autogenous Bone: An Epifluorescence Microscopy Analysis in Rats," Journal of Applied Oral Science, Vol. 18, No. 4, 2010, pp. 346-353. doi:10.1590/S1678-77572010000400005

[3] M. Li, X. Liu and B. Ge, "Calcium Phosphate Cement with BMP-2-Loaded Gelatin Microspheres Enhances Bone Healing in Osteoporosis: A Pilot Study," Clinical Orthopaedics and Related Research, Vol. 468, No. 7, 2010, pp. 1978-1985. doi:10.1007/s11999-010-1321-9

[4] Z. Lu and H. Zreiqat, "The Osteoconductivity of Biomaterials Is Regulated by Bone Morphogenetic Protein 2 Autocrine Loop Involving $\alpha 2 \beta 1$ Integrin and MitogenProtein Kinase/Extracellular Related Kinase Signaling Pathways," Tissue Engineering Part A, Vol. 16, No. 10, 2010, pp. 3075-3084. doi:10.1089/ten.tea.2010.0204

[5] P. Galindo-Moreno, M. Padial-Molina, J. E. FernándezBarbero, F. Mesa, D. Rodríguez-Martínez and F. O'Valle, "Optimal Microvessel Density from Composite Graft of
Autogenous Maxillary Cortical Bone and Anorganic Bovine Bone in Sinus Augmentation: Influence of Clinical Variables," Clinical Oral Implants Research, Vol. 21, No. 2, 2010, pp. 221-227. doi:10.1111/j.1600-0501.2009.01827.x

[6] F. Jegoux, E. Aguado, R. Cognet, O. Malard, F. Moreau, G. Daculsi and E. Goyenvalle, "Alveolar Ridge Augmentation in Irradiated Rabbit Mandibles," Journal of Biomedical Materials Research Part A, Vol. 93, No. 4, 2010, pp. 1519-1526.

[7] Y. K. Kim, S. G. Kim, S. C. Lim, H. J. Lee and P. Y. Yun, "A Clinical Study on Bone Formation Using a Demineralized Bone Matrix and Resorbable Membrane," Oral Surgery, Oral Medicine, Oral Pathology, Oral Radiology, and Endodontology, Vol. 109, No. 6, 2010, pp. 6-11. doi:10.1016/j.tripleo.2010.01.012

[8] Y. R. Kuo, H. S. Shih, C. C. Chen, R. Boca, Y. C. Hsu, C. Y. Su, et al., "Free Fibula Osteocutaneous Flap with Soleus Muscle as a Chimeric Flap for Reconstructing Mandibular Segmental Defect after Oral Cancer Ablation," Annals of Plastic Surgery, Vol. 64, No. 6, 2010, pp. 738742. doi:10.1097/SAP.0b013e3181a72f62

[9] C. T. Brighton, D. G. Lorich, T. M. Kupcha Reilly, A. R. Jones and R. A. Woodbury II, "The Pericyte as a Possible Osteoblast Progenitor Cell," Clinical Orthopaedics, Vol. 275, 1992, pp. 287-299.

[10] M. Chazono, T. Tanaka, H. Komaki and K. Fujii, "Bone Formation and Bioresorption after Implantation of Injectable Beta-Tricalcium Phosphate Granules-Hyaluronate Complex in Rabbit Bone Defects," Journal of Biomedical Materials Research Part A, Vol. 70, No. 4, 2004, pp. 542-549. doi:10.1002/jbm.a.30094

[11] J. E. Dumas, K. Zienkiewicz, S. A. Tanner, E. M. Prieto, S. Bhattacharyya and S. A. Guelcher, "Synthesis and Characterization of an Injectable Allograft Bone/Polymer Composite Bone Void Filler with Tunable Mechanical Properties," Tissue Engineering Part A, Vol. 16, No. 8, 2010, pp. 2505-2518. doi:10.1089/ten.tea.2009.0672

[12] G. Hannink, J. G. Wolke, B. W. Schreurs and P. Buma, "In Vivo Behavior of a Novel Injectable Calcium Phosphate Cement Compared with Two Other Commercially Available Calcium Phosphate Cements," Journal of Biomedical Materials Research Part B: Applied Biomaterials, Vol. 85, No. 2, 2008, pp. 478-488. doi:10.1002/jbm.b.30969

[13] T. Honma, T. Itagaki, M. Nakamura, S. Kamakura, I. Takashashi, S. Echigio and Y. Sasano, "Bone Formation in Rat Calvaria Ceases within a Limited Period Regardless of Completion of Defect Repair," Oral Diseases, Vol. 14 , No. 5, 2008, pp. 457-464. doi:10.1111/j.1601-0825.2007.01401.x

[14] J. W. Park, J. H. Jang, S. R. Bae, C. H. An and J. Y. Suh, "Bone Formation with Various Bone Graft Substitutes in Critical-Sized Rat Calvarial Defect," Clinical Oral Implants Research, Vol. 20, No. 4, 2009, pp. 372-378. doi:10.1111/j.1600-0501.2008.01602.x 